\title{
The crack growth resistance of an elastoplastic lattice
}

\author{
Harika C. Tankasala ${ }^{\mathrm{a}}$, Norman A. Fleck ${ }^{\mathrm{a}, *}$ \\ ${ }^{a}$ Department of Engineering, University of Cambridge, Cambridge CB2 1PZ, United Kingdom
}

\begin{abstract}
The degree to which the toughness of a lattice material can be enhanced by the suitable placement of multiple phases is explored. To achieve this, the resistance to mode I and mode II crack growth in a two-dimensional (2D), elastoplastic, triangulated lattice is investigated using finite element (FE) simulations. The fully triangulated lattice is idealised as a pin-jointed truss, and each strut has an axial force versus elongation (or shortening) characteristic based on the uniaxial tensile response of an elastoplastic solid with power-law hardening. When the tensile force in the strut attains a critical value, a linear softening law is adopted for the force versus elongation response of the strut to simulate its failure. FE simulations of crack growth in the 2D lattice are performed under small-scale yielding conditions, and the sensitivity of the crack growth resistance curve ( $R$-curve) to the cell wall strain hardening exponent and cell wall ductility is determined. Three concepts for enhancing the $R$-curve of a triangulated lattice are explored: (i) a brittle lattice reinforced by long ductile fibres transverse to the cracking plane, (ii) a bilattice such that a small scale brittle lattice is reinforced by a large scale ductile lattice, and (iii) a 2D version of an interpenetrating lattice wherein a large scale ductile lattice is bonded at its joints to an underlying small-scale brittle lattice.
\end{abstract}

Keywords: $R$-curve, fracture toughness, crack propagation, multi-phase, toughening mechanism, crack bridging

\footnotetext{
*Corresponding author

Email addresses: hct30@cam.ac.uk (Harika C. Tankasala), naf1@cam.ac.uk (Norman A. Fleck)
} 


\section{Introduction}

Lattice materials made from metals, polymers, and fibre-reinforced composites are finding increasing use in light-weight applications that demand high stiffness, strength, resistance to fracture, and energy absorption [1-8]. Of the many choices in topology of lattice material, the stretching-dominated arrangement (such as the triangular or Kagome configuration in 2D or the octet-truss in 3D) offers the best combination of strength, stiffness, and damage tolerance at low weight $[9,10]$. The current literature on the mechanical behaviour of lattice materials details the effective medium properties of a wide range of lattice topologies. Scaling laws have been derived for the macroscopic effective values of modulus and strength in terms of the material properties of the cell wall solid by a combination of analytical and numerical techniques [11-13]. For example, the recent study by Tankasala et al.[14] predicted the tensile response of polymeric lattices at finite strain. It is widely recognized that as-manufactured lattices suffer from imperfections such as missing/broken cell walls, wavy struts and variable strut thickness. These imperfections degrade the performance of the lattice, and the imperfection sensitivity of macroscopic stiffness and strength has been documented in both the experimental and numerical literature $[15,16]$.

In addition to stiffness and strength, the fracture response of lattice materials is of significant practical importance. Theoretical studies have explored the sensitivity of crack initiation fracture toughness $K_{C}$ to pure mode I loading, pure mode II loading, and to combined, mixed-mode loading $[12,17,18]$. These predictions of $K_{C}$ have been verified experimentally, see for example Alonso and Fleck [19] for brittle-ceramic diamond lattice, O'Masta et al. [20] for the titanium alloy octet-truss lattice, and Gu et al. [21] for the aluminium alloy triangular lattice. It remains to predict the crack growth resistance curve, the so-called $R$-curve, for a macroscopic crack in a ductile lattice or in a ductile bilattice. This is the objective of the present paper.

Our focus is on crack growth in a two-dimensional (2D) elastoplastic triangular lattice, see Fig. 1. We adopt a boundary layer approach whereby the displacement field associated with the mode I stress intensity factor $K_{\mathrm{I}}$ (or with the mode II stress intensity factor $K_{\mathrm{II}}$ ) is applied to the outer boundary of the finite element mesh. We build on the previous studies of Fleck and co-workers for the prediction of crack initiation in lattice materials using a similar boundary layer approach $[12,19,22]$. Crack advance by the sequential failure of 
struts ahead of the advancing crack-tip is also considered. A brief review of the fracture toughness of lattice materials, and crack propagation in bulk solids is first presented to motivate the present study.

A number of numerical approaches are available for modelling the fracture response of a lattice. For lattice topologies with high nodal connectivity, such as a fully triangulated lattice with 6 bars joining at a node, much insight is gained by idealising its structural response by that of a pin-jointed truss wherein each strut is under uniform stress and uniform strain states. The next level of sophistication is to idealise the lattice by rigid-jointed bars, with each bar comprising an array of Euler or Timoshenko beam elements. In general, the stress (and strain) state varies across the thickness and length of the strut, and consequently, there is choice in the criterion for crack advance. Two extreme choices have been explored in the literature for beam elements. A strut in the lattice fails when (i) the maximum local (point-wise) tensile stress (or strain) anywhere in the strut attains the solid tensile strength (or failure strain), or (ii) the mean tensile strain at any cross-section of the strut, upon averaging the axial strain over the strut thickness, attains the solid failure strain. The pointwise stress criterion is an appropriate choice for lattices made from ceramics and brittle metallic alloys which develop cracks when a maximum tensile strain is achieved [23-25]. In contrast, the average strain criterion is suitable for ductile solids which fail by necking [26]. More refined calculations of lattice behaviour may be performed in which each strut of the lattice is represented by many continuum elements. Such an approach enables assessment of the local strain distribution arising from the precise geometry of the struts and the joints between the neighbouring struts, albeit at a huge computational cost. Predictions of the fracture toughness of rigid-jointed lattices with beam elements are now reviewed.

Consider, for example, a triangular lattice comprising cell walls of thickness $t$ and length $\ell$, made from an elastic-brittle solid of fracture strength $\sigma_{\mathrm{fs}}$. The relative density $\bar{\rho}$ of the triangular lattice scales with $t$ and strut length $\ell$ as ${ }^{1}$

$$
\bar{\rho}=2 \sqrt{3} t / \ell
$$

In their numerical prediction of the fracture toughness of an elastic-brittle lattice, Romijn

\footnotetext{
${ }^{1}$ Note that the scaling law (1) over-estimates the relative density owing to the double-counting of the material at the joints. It is therefore strictly valid for low values of $t / \ell$ such as $t / \ell<0.1$. For large values of $t / \ell$, the relative density is lower by a factor of $(1-\sqrt{3} t / 2 \ell)$, see [11].
} 
and Fleck [12] and Fleck and Qiu [17] idealized each cell wall of the lattice by several Euler beam elements. A point-wise stress criterion was assumed for the failure of a critical strut at the macroscopic crack-tip. The mode I fracture toughness $K_{\text {IC }}$ of the lattice is defined as the remote mode I stress intensity factor for which the local tensile stress in a critical cell wall at the crack-tip attains the value $\sigma_{\mathrm{fs}}$. The magnitude of $K_{\mathrm{IC}}$ scales with $\sigma_{\mathrm{fs}}$, and for the triangular lattice, $K_{\mathrm{IC}}$ is given by

$$
K_{\mathrm{IC}}=0.5 \bar{\rho} \sigma_{\mathrm{fs}} \sqrt{\ell}
$$

as first reported by Romijn and Fleck [12].

In contrast, when the cell wall solid has an elastoplastic response, the macroscopic mode I fracture toughness is sensitive to the mode of cell wall failure, as discussed by Tankasala et al. [22]. They idealized each strut of the lattice by several Timoshenko beam elements, and assumed that the true stress $\sigma$ versus true strain $\varepsilon$ response of the solid material in uniaxial tension is of Ramberg-Osgood form,

$$
\frac{\varepsilon}{\varepsilon_{0}}=\frac{\sigma}{\sigma_{0}}+\left(\frac{\sigma}{\sigma_{0}}\right)^{n}
$$

in terms of a yield stress $\sigma_{0}$, yield strain $\varepsilon_{0}$ and hardening parameter $n$. The first term on the right hand side of Eq. (3) is the elastic strain (normalized by $\varepsilon_{0}$ ) while the second term is the plastic strain (again normalized by $\varepsilon_{0}$ ). The Young's modulus of the solid $E_{\mathrm{S}}$ equals $\sigma_{0} / \varepsilon_{0}$. Tankasala et al. [22] made predictions of $K_{\mathrm{IC}}$ for a range of lattice topologies by assuming that failure occurs in a critical strut at the crack-tip of the lattice when (i) the maximum point-wise tensile strain attains the cell wall failure strain, $\varepsilon_{\mathrm{f}}$, or (ii) the average tensile strain across the strut attains the value $\varepsilon_{\mathrm{f}}$. The sensitivity of $K_{\mathrm{IC}}$ to these alternative failure criteria was found to be mild for stretching-dominated lattices such as the triangular lattice. $K_{\mathrm{IC}}$ scales with $\bar{\rho}$ and $\ell$ in the same manner as that stated in (2), but now also depends upon the degree of material strain hardening $n$ and upon $\varepsilon_{\mathrm{f}}$. They found that the critical fracture toughness $K_{\mathrm{IC}}$ for the elastoplastic triangular lattice scales as

$$
\mathrm{K}_{\mathrm{IC}} \approx 0.5 \bar{\rho}\left(\frac{\varepsilon_{\mathrm{f}}}{\varepsilon_{0}}\right)^{\frac{n+1}{2 n}} \sigma_{0} \sqrt{\ell}
$$

Further, the extent $r_{\mathrm{P}}$ of the plastic zone ahead of the crack-tip, at a given value of stress intensity factor $K_{\mathrm{I}}$, scales with the lattice relative density $\bar{\rho}$ and cell wall yield strength $\sigma_{0}$ as

$$
r_{\mathrm{P}}=\alpha\left(\frac{K_{\mathrm{I}}}{\sigma_{0} \bar{\rho}}\right)^{2}
$$


The constant $\alpha$ depends upon $n$ such that $\alpha$ equals $1.4,1.7$, and 2.6 for $n$ equal to 3,10 , and $\infty$, respectively. As the crack-tip is approached, within this plastic zone, the macroscopic stress and macroscopic strain approach the $J$-field for a dilatant plastic solid. This asymptotic field is a variant of the well-known HRR field for an isotropic von Mises solid with finite strain hardening.

Little is known about crack growth in a lattice material made from an elastoplastic solid, and so, by way of background, we briefly review crack advance in the fully dense, parent solid. Under conditions of small-scale yielding, crack growth in a fully dense ductile solid is generally of the form

$$
K=K_{\mathrm{R}}(\Delta a)
$$

where the $K_{R}(\Delta a)$ curve is treated as a material characteristic, termed the $R$-curve. It is broadly accepted that, for ductile fracture, $K_{R}(\Delta a)$ rises steeply above the initiation value $K_{\mathrm{IC}}$ due to the effects of crack-tip plasticity and non-proportional loading in the wake of the advancing crack-tip. Many of these features of ductile crack growth have been captured by the cohesive zone model of Tvergaard and Hutchinson [27]. Their analysis suggests that the $R$-curve is sensitive to the ratio of peak cohesive strength to the yield strength of the solid.

A limited literature exists on crack growth in a lattice material. In an early numerical study on the crack growth resistance of metallic foams, Chen et al. [28] idealised the foam as a compressible, elastoplastic, power-law hardening solid. They determined the crack growth resistance of a semi-infinite crack in the foam by placing a cohesive zone at the crack-tip in similar manner to that of Tvergaard and Hutchinson [27], and they calibrated the crack-bridging law from independent experimental measurements on these foams. The predictions of Chen et al. [28] for the crack growth resistance curves reveal a pronounced $R$-curve: the advancing crack experiences a rapidly increasing resistance to its growth, with the extent of $R$-curve sensitive to the compressibility of the metal foam. They found that the asymptotic steady-state mode I fracture toughness increases with increasing compressibility of the foam. In a parallel study, Schmidt and Fleck [29] simulated crack growth in a 2D hexagonal lattice comprising cell walls made from a bi-linear elastoplastic solid. Crack growth in their FE model was simulated by deleting beam elements that had attained the cell wall strength. Failure of these elements was achieved by assuming a local soften- 
ing response: nodal loads were gradually reduced to zero in accordance with a specified work of fracture. The resulting $K$ versus $\Delta a$ resistance curves were sensitive to the choice of hardening modulus and to the fracture strength of the parent solid, and an appreciable elevation in the $R$-curve was noted. In a recent experimental study, O'Masta et al. [20] measured the fracture response of an octet truss lattice made from a titanium alloy and also observed a rising $R$-curve during crack propagation, akin to that of a bulk solid.

The present study considers crack propagation in a elastoplastic triangular lattice made from a power-law hardening solid of assumed tensile failure strain ${ }^{2}$. The choice of this topology is motivated by the fact that the triangular lattice has a high fracture toughness $K_{\mathrm{IC}}$ due to its high nodal connectivity and is thus a promising $2 \mathrm{D}$ lattice. Its fracture response also gives insight into the response of 3D lattices of high nodal connectivity, such as the octet truss.

The finite element models of triangular lattices of the present study contain up to $10^{7}$ struts in order to model a remote $K$-field and an inner $J$-field for crack extensions of up to $50 \ell$. It was prohibitive, computationally, to model each strut by a large number of continuum elements. Additionally, the continuum description of a lattice (eg. [30]) can be problematic as sudden changes in section at a joint can introduce singularities in local stress. Such local features would require a sophisticated, multi-scale approach for their treatment; this approach is beyond the scope of the present study. Instead, we note that the triangular lattice is stretching-dominated under all macroscopic stress states due to its high nodal connectivity. The structural response of a pin-jointed (truss) of high nodal connectivity, such as the triangular lattice, is in close agreement with that of the rigid-jointed lattice (frame), see for example [31, 32]. The aforementioned features of triangular lattice permit its idealization by a pin-jointed truss, thereby enabling the use of a single finite element per strut.

\footnotetext{
${ }^{2}$ The necking strain $\varepsilon_{\mathrm{n}}$ of a bar of perfect geometry follows from Considére criterion as $\varepsilon_{\mathrm{n}}=1 / n$. The presence of local geometric imperfections in the bar leads to a softening response at values of strain less than $1 / n$. In the current study, we explore the sensitivity of the lattice response to failure strain below the necking value.
} 


\subsection{Scope of study}

Mode I crack propagation in a elastoplastic triangular lattice is studied under small scale yielding conditions. The crack growth resistance curve ( $R$-curve) of the lattice is predicted from finite element (FE) simulations. An asymptotic $K$-field approach is adopted, and crack growth within the lattice occurs by the progressive failure of struts under a monotonically increasing remote $K_{\mathrm{I}}$. Denote $K_{\mathrm{R}}$ as the remote value of $K_{\mathrm{I}}$ corresponding to a crack extension $\Delta a$. Then, the $R$-curve of the lattice is the $K_{R}(\Delta a)$ response.

Our study is organized as follows. First, the $R$-curve for an elastoplastic triangular lattice under mode I loading is predicted via an asymptotic $K$-field boundary layer approach. The sensitivity of both the $R$-curve and the crack path to the choice of cell wall material properties such as the ductility and extent of strain hardening, along with the relative density of the lattice, is explored by FE simulations. A regression analysis is performed to provide an expression for the $R$-curves in terms of the solid material properties.

Next, three design concepts for enhancing the crack growth resistance of a triangular lattice are explored: (i) a fibre-reinforced lattice, comprising a brittle lattice phase and reinforced by long ductile fibres transverse to the plane of the pre-crack, (ii) a bilattice, generated by reinforcing a small brittle lattice with a large ductile lattice, and (iii) an interpenetrating lattice wherein a large-scale ductile lattice is attached at its joints to an underlying small-scale brittle lattice. In each case, the predicted $R$-curves are compared with those of single phase lattices to highlight the role of ductile reinforcements on the $R$-curve. Finally, a comparative study is performed to analyse the crack growth resistance of single-phase elastoplastic lattices under remote mode II loading.

\section{Numerical approach}

The crack growth resistance of an elastoplastic triangular lattice is determined via a boundary layer analysis within the finite element (FE) framework. Quasi-static FE calculations are performed with ABAQUS/Explicit v6.14 to simulate crack growth in an elastoplastic lattice. The FE mesh of the pin-jointed triangular lattice comprises struts of length $\ell$ in a square domain of edge length $1600 \ell$. The pre-crack spans $800 \ell$, and the tip of the crack is located at the centre of the square mesh, as shown in Fig. 1. It is assumed that the pre-crack splits 
the joints on the cracking plane, behind the crack tip. Each strut of the lattice is modelled by a single two-noded truss element (type T2D2) which can only carry an axial load. The outer boundary of the FE mesh is subjected to the asymptotic displacement field associated with the mode I stress intensity factor $K_{\mathrm{I}}$. Crack advance is by failure of struts (and not joints) using a Johnson-Cook type damage model ${ }^{3}$ for each strut. The size of the FE mesh is sufficient for small scale yielding conditions to prevail for an extension $\Delta a \leq 50 \ell$. The FE simulations assumed finite deformations and finite strains. Details of the boundary layer approach and the assumed material model are presented below.

\subsection{Boundary layer method}

Consider a semi-infinite crack in a triangular lattice with cell walls of power-law hardening characteristic, and loaded by a remote mode I $K$-field. The crack is along the negative $x_{1}$ axis with its tip at the origin $\left(x_{1}, x_{2}\right)=(0,0)$, as shown in Fig. 1 . Write the displacement field in Cartesian form as $u_{i}\left(x_{j}\right)$, and introduce the polar coordinate system $(r, \theta)$ centred on the crack-tip, with the crack faces lying along $\theta= \pm \pi$. Here, $(r, \theta)$ are related to $\left(x_{1}, x_{2}\right)$ in the usual manner as $r=\left(x_{1}^{2}+x_{2}^{2}\right)^{1 / 2}$ and $\theta=\tan ^{-1}\left(x_{2} / x_{1}\right)$. The displacement field in an elastic annulus surrounding the crack-tip plastic zone scales with $K_{\mathrm{I}}$ according to

$$
u_{i}^{0}=\frac{K_{\mathrm{I}} \sqrt{r}}{G} f_{i}(\theta)
$$

where $G$ is the shear modulus of the triangular lattice written in terms of Young's modulus $E$ and Poisson's ratio $v$ as $G=E / 2(1+v)$ where $E=\bar{\rho} E_{S} / 3$ and $v=1 / 3$, as stated by Gibson and Ashby [11]. The non-dimensional function $f_{i}(\theta)$ is taken from Sih et al. [33]. The linear scaling of $E$ with $\bar{\rho}$ is limited to the regime where the struts can be idealized as truss elements such that $t / \ell \leq 0.2$. Note that $t / \ell=0.1$ corresponds to $\bar{\rho}=0.34$ in a $2 \mathrm{D}$ triangular lattice and it corresponds to $\bar{\rho}=6 \sqrt{2}(t / \ell)^{2}=0.08$ for a 3D octet truss lattice. Consequently, the use of truss elements is pertinent to much higher values of $\bar{\rho}$ in a 2D lattice than in a 3D lattice.

As the value of $K_{\mathrm{I}}$ is increased, a plastic zone develops at the crack-tip and envelopes an increasing number of cells, recall (5). A critical strut within the plastic zone and directly ahead of the crack-tip attains the cell wall tensile failure strain $\varepsilon_{\mathrm{f}}$ at $K_{\mathrm{I}}=K_{\mathrm{IC}}$, as given by

\footnotetext{
${ }^{3}$ The Johnson-Cook ductile damage model within ABAQUS is used with parameters to give a strain-rate independent and temperature-independent strain criterion for damage initiation.
} 
(4). Progressive failure of the strut then follows, in accordance with a specified damage law (described below in Section 2.2). A sequence of struts fail under an increasing value of $K_{\mathrm{I}}$, thereby leading to a rising $R$-curve.

\subsection{Material model}

Each strut of the lattice is specified with an axial force versus elongation response. The axial tensile and compressive responses of each strut are based upon the uniaxial tensile response of an elastoplastic, von Mises solid with power-law hardening. When the tensile force in the strut attains a critical value, a linear softening law is assumed for the force versus elongation response of the strut, in accordance with a prescribed work of fracture. In contrast, the compressive response of the strut follows the assumed power-law hardening behaviour for all values of compressive force; it is assumed that no buckling occurs ${ }^{4}$. The axial stress and strain state is taken to be uniform within each strut, and so the assumed force versus elongation response during tensile failure is associated with a softening response of the strut material. It is recognized that a softening law at the material level would lead to localization at some location within the strut, but this is not at issue here since the stress and strain states are assumed uniform, and merely express the strut force versus elongation response as re-scaled values. Details of the implementation of this material model are as follows:

(i) The initial response of the cell wall material is characterized by the solid Young's modulus $E_{\mathrm{S}}$ and Poisson's ratio $v_{\mathrm{S}}$.

(ii) The post-yield response of the solid is idealized by a power-law relation between the true stress $\sigma$ and the true plastic strain $\varepsilon^{\mathrm{P}}$ of the form

$$
\frac{\sigma}{\sigma_{0 S}}=1+\left(\frac{\varepsilon^{\mathrm{P}}}{\varepsilon_{0 \mathrm{~S}}}\right)^{N}
$$

in terms of the material flow strength $\sigma_{0 \mathrm{~S}}$ and a representative material strain $\varepsilon_{0 \mathrm{~S}}$. The index $N$ is the Ludwik/Holloman strain hardening exponent. We emphasize that the two relations (3) and (8) can be brought into alignment at a sufficiently large value of plastic strain upon identifying $\sigma_{0}=\sigma_{0 S}, \varepsilon_{0}=\varepsilon_{0 S}$ and $N=1 / n$.

\footnotetext{
${ }^{4}$ The possibility of occurrence of buckling at the crack tip of a fully triangulated lattice was included in the previous analysis of Tankasala et al. [14]. No buckling was observed.
} 
(iii) Damage initiates within the strut when the tensile plastic strain in the strut, $\varepsilon^{\mathrm{P}}$, attains a critical value $\varepsilon_{\mathrm{f}}$, or equivalently, a strength $\sigma_{\mathrm{f}}$ as indicated in Fig. 2(a).

(iv) Damage evolves within the strut as follows. The axial stress $\sigma$ in the strut drops linearly from $\sigma_{\mathrm{f}}$ to zero over a plastic strain increment of $\Delta \varepsilon_{\mathrm{f}}$, see Fig. 2(a). Write $\Gamma_{0}$ as the work of fracture, per unit cross-sectional area of strut, in the softening regime. Then, $\Gamma_{0}=\sigma_{\mathrm{f}} \Delta \varepsilon_{\mathrm{f}} \ell / 2$ in terms of the strut length $\ell$. The plastic work of fracture per unit cross-sectional area of the strut $\Gamma_{\mathrm{f}}$ follows as

$$
\Gamma_{\mathrm{f}}=\ell \int_{0}^{\varepsilon_{\mathrm{f}}} \sigma d \varepsilon^{\mathrm{P}}+\Gamma_{0}
$$

(v) Some struts in the wake of the advancing crack-tip unload but remain intact. The unloading modulus $E_{\mathrm{u}}$ of these struts depends upon the value of instantaneous plastic strain $\varepsilon^{\mathrm{P}}$ as follows: $E_{\mathrm{u}}=E_{\mathrm{S}}$ for $\varepsilon^{\mathrm{P}} \leq \varepsilon_{\mathrm{f}}$ and $E_{\mathrm{u}}=(1-D) E_{\mathrm{S}}$ for $\varepsilon_{\mathrm{f}} \leq \varepsilon^{\mathrm{P}} \leq \varepsilon_{\mathrm{f}}+\Delta \varepsilon_{\mathrm{f}}$ where the damage index $D$ is defined by $D=\left(\varepsilon^{\mathrm{P}}-\varepsilon_{\mathrm{f}}\right) / \Delta \varepsilon_{\mathrm{f}}$. Note that $D$ increases from zero (no damage) to unity (failure). We note in passing that the choice of $E_{\mathfrak{u}}\left(\varepsilon^{\mathrm{P}}\right)$, along with assumption (iv) above implies that the elastic strain is constant and equals $\sigma_{\mathrm{f}} / E_{\mathrm{S}}$ during the softening phase. Thus, a linear drop in $\sigma\left(\varepsilon^{\mathrm{P}}\right)$, as shown in Fig. 2(a), also gives a linear drop in $\sigma(\varepsilon)$ where the total strain $\varepsilon$ is the sum of the elastic and plastic components.

The tensile stress versus strain response of the cell wall based on the above description is shown in Fig. 2(b) for $\varepsilon_{\mathrm{f}}=0.1, \varepsilon_{0 \mathrm{~S}}=0.001$ and for selected values of $N$. In order to visualise the linear softening regime in the figure, we show the softening curves for the choice $\bar{\Gamma}=\Gamma_{0} E_{S} / \sigma_{0 S}^{2} \ell=50$. It is emphasized that the response of the lattice during crack growth is almost insensitive to the value of $\Gamma_{0}$. The softening phase leads to a mild snapback dynamic instability near the crack tip when $\Gamma_{0}$ is below a critical value $\Gamma_{\mathrm{c}}$. However, we find that this instability is quickly damped by a small level of plastic dissipation in the neighbouring struts to the failed strut, as detailed below. The value of $\Gamma_{\mathrm{c}}$ is dependent upon the unloading modulus $\sigma_{\mathrm{f}} / \Delta \varepsilon_{\mathrm{f}}$ of the failing strut in relation to the modulus of the neighbouring intact, but plastically deforming, bars. The choice $\Gamma_{0}=\Gamma_{\mathrm{c}}$ would give rise to a quasi-static solution with no generation of kinetic energy during the failure of the strut. Checks have been made to determine the value of $\Gamma_{\mathrm{c}}$ for selected crack growth simulations. The following numerical device was used in order to estimate $\Gamma_{\mathrm{c}}$. In brief, a subsidiary 
implicit finite element calculation was performed with the remote $K$ set to a constant value $K_{\mathrm{f}}$ during strut failure. Here, $K_{\mathrm{f}}$ is the mode I stress intensity factor corresponding to the onset of softening i.e. the attainment of $\sigma=\sigma_{\mathrm{f}}$ in the first critical strut ahead of the crack tip, recall Fig. 2(a). The softening response of this critical strut was now modified by the introduction of a monotonically increasing thermal strain $\varepsilon_{T}$ within this strut, while $\varepsilon^{\mathrm{P}}$ was held fixed at $\varepsilon_{\mathrm{f}}$, such that

$$
\sigma=E_{\mathrm{S}}\left(\varepsilon-\varepsilon_{\mathrm{f}}-\varepsilon_{\mathrm{T}}\right)
$$

in terms of a total strain $\varepsilon$ and elastic modulus of the cell wall solid $E_{\mathrm{S}}$. This replaces the softening branch of Fig. 2(a). The thermal strain $\varepsilon_{\mathrm{T}}$ is treated as an independent control parameter within $\mathrm{ABAQUS}$, and is incremented until the axial stress in the strut drops to zero in the finite element calculation; write the final value of $\varepsilon_{\mathrm{T}}$ as $\varepsilon_{\mathrm{Tf}}$. It was found that the $\sigma\left(\varepsilon^{\mathrm{P}}\right)$ response is almost linear during the softening phase. At vanishing axial stress, $\sigma=0$, the axial strain in the critical strut equals $\varepsilon_{\mathrm{f}}+\varepsilon_{\mathrm{Tf}}$ by (10). Now, compare this residual strain with the plastic strain $\left(\varepsilon_{\mathrm{f}}+\Delta \varepsilon_{\mathrm{f}}\right)$ of Fig. 2(a), to note that $\Delta \varepsilon_{\mathrm{f}}=\varepsilon_{\mathrm{Tf}}$ in order to bring the two calculations into alignment. We emphasise that $\sigma(\varepsilon)$ during the thermal softening event of the critical strut, as determined by the finite element solution, is almost linear. Further, the Johnson-Cook type softening model of Fig. 2(a) implies a linear $\sigma(\varepsilon)$ softening characteristic. Consequently, the thermal calculation is a match to the prediction of the damage model, provided we take $\Gamma_{\mathrm{c}}=\sigma_{\mathrm{f}} \mathcal{E}_{\mathrm{Tf}} \ell / 2$.

The assumed value of $\Gamma_{0}$ in the FE simulations of crack growth is typically an order of magnitude less than $\Gamma_{\mathrm{c}}$. The choice of $\Gamma_{0}=0.1 \Gamma_{\mathrm{c}}$ would give rise to a small snap-back instability in a quasi-static finite element simulation [34]. In the explicit simulations, the instability induces a small amount of kinetic energy and plastic dissipation in the struts adjacent to the failed strut. The energy released by the strut during failure (with our assumed value of $\Gamma_{0}$ ) is two orders of magnitude less than the plastic work in a strut up to the point of failure. Consequently, the additional plastic dissipation in the neighbouring struts is negligible and there was no observable difference in the two $R$-curves for the choices $\Gamma_{0}=\Gamma_{\mathrm{c}}$ and $\Gamma_{0}=0.1 \Gamma_{\mathrm{c}}$. 


\section{3. $R$-curve of an elastoplastic triangular lattice}

The FE predictions for the $R$-curves of a triangular lattice of $t / \ell=0.1$ made from a solid of $\varepsilon_{0 S}=0.001, v_{S}=0.3, \bar{\Gamma}=5$, and $N=0.33$ are plotted in Fig. 3(a) for selected values of $\varepsilon_{\mathrm{f}}$ in the range 0.02 to 0.2 . The abscissa for these plots is the normalized crack extension $\Delta a / \ell$ at a given value of $K_{\mathrm{I}}$, as plotted on the ordinate. Each cross mark in Fig. 3(a) denotes the $x_{1}$-position of the mid-point of all failed struts at a given value of $K_{\mathrm{I}}=K_{\mathrm{R}}$, with the rightmost cross-mark (corresponding to the furthest failed strut from the pre-crack tip) denoting the crack extension $\Delta a$.

The first strut to fail is the vertical strut, strut A, directly ahead of the crack tip, refer inset of Fig. 3(d). Its failure defines the onset of crack growth and thereby the initiation fracture toughness of the lattice, $K_{\mathrm{IC}}$. For the choice $\bar{\Gamma}=5$, we note from Fig. 3(a) that $K_{\mathrm{IC}}$ is within $5 \%$ of the predictions of (4) for $\varepsilon_{\mathrm{f}}$ between 0.02 and 0.2 . Consider, for example, the case of $\varepsilon_{\mathrm{f}}=0.1$. The second strut to fail is the vertical strut, strut $\mathrm{B}$, nearest to the failed strut $\mathrm{A}$; it fails at $K_{\mathrm{R}} / \sigma_{0 S} \sqrt{\ell}=5$. Post failure of the first two vertical struts (A and B), the inclined strut located between these struts rotates to align with the loading direction and it eventually fails in tension at a higher value of $K_{R} / \sigma_{0 S} \sqrt{\ell}=5.4$. Crack advance is thus accompanied by a bridging of the cracking plane by the inclined struts. The solid lines in Fig. 3(a) are conservative curve-fit estimates to the data for each value of $\varepsilon_{\mathrm{f}}$ between 0.02 and 0.2 ; these have the form

$$
K_{\mathrm{R}}=K_{\mathrm{IC}}+\alpha_{1} \bar{\rho} \sigma_{0 \mathrm{~S}} \sqrt{\ell}\left(\frac{\Delta a}{\ell}\right)^{\alpha_{2}}
$$

The parameters $\alpha_{1}$ and $\alpha_{2}$ depend on $\left(N, \varepsilon_{\mathrm{f}}\right)$ and are listed in Table 1. It is evident from Fig. 3(a) that the ductility of cell wall solid has a significant effect on the crack growth resistance such that the magnitude of $K_{R}$ and of the gradient $\partial K_{R} / \partial \Delta a$ increase with increasing $\varepsilon_{\mathrm{f}}$. Consider, for example, a crack extension of $\Delta a=20 \ell$. The associated fractional increase in the fracture toughness, $K_{\mathrm{R}}(\Delta a) / K_{\mathrm{IC}}$ is 1.3 for $\varepsilon_{\mathrm{f}}=0.02$, and is $K_{\mathrm{R}}(\Delta a) / K_{\mathrm{IC}}=3$ for $\varepsilon_{\mathrm{f}}=0.2$. The reference case of a triangular lattice made from an elastic-brittle cell wall solid is included in Fig. 3(a), with $\sigma_{0 S}$ now denoting the solid tensile strength. Brittle failure of the lattice occurs when $K_{\mathrm{I}} \approx K_{\mathrm{IC}}$, with a negligible increase in the fracture toughness as the crack grows: the $R$-curve is flat. The nature of crack advance is demonstrated in Fig. 3(d) by considering the tip opening displacement of the pre-crack, measured at location $c$, one bar back from strut $A$, for the choice of $\varepsilon_{\mathrm{f}}=0.1$. Immediately after failure of 
strut $\mathrm{A}$, and then strut $\mathrm{B}$, the opening displacement $\delta_{c}$ continues to increase in a smooth manner with increasing $K_{R}$. The softening of struts A or B does not trigger substantial oscillations in the crack tip opening response.

The sensitivity of the $R$-curve to the degree of material strain hardening is explored in Fig. 3(b) for $N=0.1,0.2$, and 0.33 , and for a fixed $\varepsilon_{\mathrm{f}}=0.1$ and $t / \ell=0.1$. As before, the cross marks correspond to the $x_{1}$-position of the mid-points of all struts that have failed at a given value of $K_{R}$, and the solid lines are the curve-fit expressions (11) with values of $\alpha_{1}$ and $\alpha_{2}$ as listed in Table 1 . The $R$-curve is steepest for lattices made from material of high strain hardening consistent with the behaviour of fully dense metallic alloys, see [27] for example.

The $R$-curve asymptotes to a steady-state tearing value of fracture toughness $K_{\mathrm{R}}=K_{\mathrm{SS}}$ at sufficiently large values of crack extension $\Delta a$. For example, for the choice $\varepsilon_{\mathrm{f}}=0.02$ and $N=0.33$, steady-state crack growth occurs for crack extensions exceeding $30 \ell$ and $K_{\mathrm{SS}}$ attains the value of $1.5 K_{\mathrm{IC}}$. The amount of crack extension in order to achieve steady state increases with increasing $\varepsilon_{\mathrm{f}}$ and increasing $N$, see Fig. 3(a) and Fig. 3(b). These simulations of crack growth under small-scale yielding are numerically intensive and limitations in computational resource precluded us from attaining steady state at large $\varepsilon_{\mathrm{f}}$ and large $N$.

For a given $\varepsilon_{\mathrm{f}}$ and $N$, the normalised fracture toughness $K_{\mathrm{IC}} / \sigma_{0 S} \sqrt{\ell}$ and the crack growth resistance $K_{\mathrm{R}} / \sigma_{0 S} \sqrt{\ell}$ (at a given value of $\Delta a / \ell$ ) scale linearly with $t / \ell$, as seen from Fig. 3(c); this follows immediately from the assumption that the cell walls behave as truss elements.

\subsection{Explanation for the rising $R$-curve, and the crack path}

The elevation in $K_{\mathrm{R}}$ with increasing $\varepsilon_{\mathrm{f}}$ (and increasing $N$ ), as observed in Fig. 3(a) and Fig. 3(b) for the ductile lattices, can be traced to the plastic dissipation associated with non-proportional stressing of the struts in the vicinity of the advancing crack-tip, which in turn relates to the extent of plastic zone at the crack-tip. Recall from (4) and (5) that the size of plastic zone $r_{\mathrm{P}}$ at the onset of crack growth scales with $\left(N, \varepsilon_{\mathrm{f}}\right)$ according to

$$
r_{\mathrm{P}} \approx 0.25 \alpha\left(\frac{\varepsilon_{\mathrm{f}}}{\varepsilon_{0}}\right)^{1+N} \ell
$$

Thus, for a given choice of $N=0.33$ and $\varepsilon_{0}=\varepsilon_{0 S}=0.001$, the extent of plastic zone, and thereby the energy dissipated due to plastic deformation increases with increasing $\varepsilon_{\mathrm{f}}$. For 
example, $r_{\mathrm{P}}$ increases from $19 \ell$ to $402 \ell$ as $\varepsilon_{\mathrm{f}}$ increases from 0.02 to 0.2 , at the onset of crack growth, following (12).

FE simulations reveal that crack advance under remote mode I loading may deviate from the plane of the pre-crack. Three classes of crack path can be identified in Fig. 4:

(i) co-planar with the pre-crack for lattices of sufficiently high ductility or of moderate hardening capacity. For example, crack advance is of this type for a lattice of high ductility $\varepsilon_{\mathrm{f}}=0.2$, and for a lattice of moderate hardening $N=0.2$ (and also for $N=0.1$, not shown).

(ii) an alternating crack path for an intermediate value of $\varepsilon_{\mathrm{f}}$ and large $N$. The wavelength (and amplitude) of the alternating crack path increases with diminishing value of $\varepsilon_{\mathrm{f}}$ and increases with crack extension. Consider the choice $\varepsilon_{\mathrm{f}}=0.1$ : the crack-tip advances along a zigzag path for $N=0.33$ but advances along a straight path (coplanar with pre-crack plane) for $N \leq 0.2$.

(iii) the crack kinks at an angle $-30^{\circ}$ to the plane of the pre-crack in the elastic-brittle limit.

It is instructive to compare the present results with the crack path predictions of Schmidt and Fleck [29] for a 2D hexagonal lattice made from a bi-linear elastoplastic solid. They also observed that, subsequent to the first strut failure, the initial symmetry about the plane of the pre-crack is disrupted and the crack advanced in a zigzag manner.

\section{Two-phase lattices for enhanced crack growth resistance}

Design concepts for enhancing the toughness of a lattice are now explored. Three designs of a two-phase lattice are considered: a fibre-reinforced composite lattice, a composite bilattice and an interpenetrating lattice, see Fig. 5. The topologies of the fibre-reinforced lattice and bilattice are inspired by the design of rip-stop nylon, a tear-resistant woven fabric made from nylon and consisting of thick reinforcement threads spaced evenly after every 10 thin fibres. The interpenetrating topology resembles that of double network hydrogels which derive their high toughness from the synergy of two mechanisms: crack 
bridging by the larger network of covalent crosslinks, and hysteresis due to unzipping of the smaller network of ionic crosslinks [35].

All three lattices comprise a 'brittle' phase of tensile failure strain $\varepsilon_{\mathrm{f}}=0.02$ and a 'ductile' phase of ductility $\varepsilon_{\mathrm{f}}=0.1$. The elastic and plastic properties of the two phases are otherwise identical such that $N=0.33, \varepsilon_{0 S}=0.001, v_{S}=0.3$ and $\bar{\Gamma}=5$. In all three designs of two-phase lattices, the brittle phase comprises a fully triangulated lattice of strut length $\ell$ and strut thickness $t$. The ductile phase is also of thickness $t$ but is on a larger grid, as follows. Consider first, the fibre-reinforced composite lattice; it comprises longitudinal ductile fibres at a spacing $s=3 \sqrt{3} \ell / 2$, as shown in Fig. 5(a). These fibres replace the brittle phase of the same location. Second, consider the composite bilattice, as shown in Fig. 5(b). The ductile phase exists on a triangular grid of side length $3 \ell$ and again substitutes for the brittle phase at this location. In contrast, the interpenetrating lattice comprises a triangular grid of ductile struts that are in addition to, and overlap, the brittle phase: no brittle struts are replaced by the ductile phase, see Fig. 5(c). The effective Young's modulus and Poisson's ratio of the interpenetrating lattice are $E=8 \sqrt{3} E_{S} t / 9 \ell$ and $v=1 / 3$, respectively, as obtained from separate FE simulations on a periodic unit cell of this lattice (not shown here). The $R$-curve responses of the three two-phase lattices are discussed in turn.

\section{1. $R$-curve of a fibre-reinforced composite lattice}

Consider the fibre-reinforced composite lattice of Fig. 5(a). The assumed morphology of the macroscopic pre-crack is such that a ductile strut of the fibre phase exists directly ahead of the crack-tip. Crack growth in this lattice occurs by the sequential failure of both brittle and ductile struts under increasing $K_{\mathrm{I}}$. The $R$-curve response of the fibre-reinforced lattice, based on the failure of brittle struts, is compared with that of the monolithic brittle lattice (of $\varepsilon_{\mathrm{f}}=0.02$ ) in Fig. 6(a). The $R$-curve for the brittle phase of the fibre-reinforced lattice lies above that of the monolithic brittle lattice. Note that the extent of crack extension $\Delta a$ in the ductile phase of the fibre-reinforced lattice is less than that of the brittle phase for the same value of $K_{\mathrm{I}}=K_{\mathrm{R}}$. Consequently, we can identify an $R$-curve for the ductile fibre phase which is distinct from that for the brittle phase.

A set of additional calculations have been performed in order to determine the sensitivity of the $R$-curves to the state of residual stress within the lattice. Commonly, thermal 
processes are used in composite manufacture and these can result in either tensile or compressive residual stress $\sigma_{\mathrm{R}}$ in the fibre phase. To assess the significance of the residual stress upon the $R$-curves, the $K$-field boundary layer analysis is carried out on a lattice specimen in which the ductile fibres carry a residual tensile (or compressive) stress. A three-step FE analysis is adopted, as follows. In the first step, the lattice does not contain a crack and a uniform state of residual stress in the ductile phase is generated by subjecting the ductile struts to thermal strain such that, at equilibrium, the ductile fibres have a residual tensile stress of magnitude $\sigma_{\mathrm{R}}=\sigma_{0 S} / 2$ whereas the vertical struts of the brittle phase have a residual compressive stress of magnitude $\sigma_{\mathrm{R}} / 2$. The inclined struts of the brittle phase remain stress free. In the second step of this FE simulation, a pre-crack is introduced. The pre-crack splits the joints along the crack flanks, and causes some relaxation of the tensile stress in the ductile bars, and the development of axial compression in the adjacent inclined bars. Likewise, the axial compressive stress in the brittle bars partially relaxes and the adjacent inclined bars acquire tension. Finally, in the third step, a remote displacement field associated with a mode I $K$-field is imposed on outermost boundary of the mesh.

\section{2. $R$-curve of a bilattice}

Assume a macroscopic pre-crack in a bilattice such that a ductile strut exists directly ahead of the crack-tip, as shown in Fig. 5(b). The sequence of strut failure with increasing $K_{\mathrm{I}}$ is plotted in Fig. 6(b) for both the brittle phase and the ductile phase of the bilattice; both axes are expressed in terms of the strut length $\ell$ of the brittle phase. In order to assess the increase in crack growth resistance due to the presence of the ductile reinforcement, we include in Fig. 6(b) the $R$-curve of a small brittle lattice with $\varepsilon_{\mathrm{f}}=0.02$ and strut length $\ell$. We note from Fig. 6(b) that the small brittle lattice is toughened when a fraction of its members are replaced by ductile struts in the bilattice. For this bilattice, the first ductile strut to fail does so after 8 vertical struts of the brittle phase have already failed. Thus, the ductile struts provide significant bridging of the crack faces in the wake of failed brittle struts, for the bilattice. This contributes to the observed resistance to crack growth, in addition to the contribution from crack-tip plasticity ahead of the growing crack-tip.

Now compare in Fig. 6(b) the $R$-curve of the bilattice with the $R$-curve of a large ductile lattice of $\varepsilon_{\mathrm{f}}=0.1$ and strut length $3 \ell$. In so doing, the significance of the small brittle struts of the bilattice upon its $R$-curve can be assessed. It is clear that the brittle phase 
gives an initial enhancement of toughness of the ductile phase of the bilattice but the two $R$-curves converge at a large crack extension, $\Delta a>40 \ell$. In broad terms, the $R$-curve of the bilattice is dominated by the contribution of the large-scale ductile phase.

\section{3. $R$-curve of an interpenetrating lattice}

The $R$-curve of the interpenetrating lattice of Fig. 5(c) is shown in Fig. 6(c) for the choice $t / \ell=0.1, N=0.33$ and $\varepsilon_{0 S}=0.001$. Two reference case of single-phase lattices are included in Fig. 6(c) for comparison: (i) a small brittle lattice of strut thickness $t$, strut length $\ell$ and $\varepsilon_{\mathrm{f}}=0.02$, and (ii) a large ductile lattice of strut thickness $t$, strut length $3 \ell$ and $\varepsilon_{\mathrm{f}}=0.1$. The $R$-curve of the brittle phase of the interpenetrating lattice is steeper than that of the single-phase brittle lattice. Further, the value of $K_{\mathrm{I}}$ required to break a single strut of the ductile phase of the interpenetrating lattice is substantially higher than that of the brittle phase, as well as that of the single-phase large ductile lattice. The crack growth resistance of the interpenetrating network is thus a consequence of limited plasticity within the small lattice and bridging of the crack-tip by the ductile struts of the large lattice.

It is instructive to compare the degree of fracture toughness enhancement due to the addition of a ductile phase for the three designs discussed above. The $R$-curves of the brittle phase of the two-phase lattices are taken from Fig. 6 and are re-plotted in Fig. 7(a); it reveals that the interpenetrating network gives the greatest enhancement of toughness. Note that the interpenetrating lattice is of higher relative density than the fibre-reinforced lattice and the bilattice for the same value of $t / \ell: \bar{\rho}=(8 / \sqrt{3}) t / \ell$ for the interpenetrating lattice and $\bar{\rho}=2 \sqrt{3} t / \ell$ for the other two topologies. In order to compare the performance of the three two-phase lattices on the basis of equal mass, we exploit the linear scaling of $K_{\mathrm{R}}$ with $t / \ell$ for each lattice and re-plot the $K_{\mathrm{R}}-\Delta a$ curves in Fig. $7(b)$, all for $\bar{\rho}=0.1$. We conclude that the superior performance of the interpenetrating lattice to that of the other two reinforced lattices is maintained at sufficiently large $\Delta a>20 \ell$.

\section{Comparison of the crack growth resistance under mode I and mode II loading}

The boundary layer approach of Section 2 can be extended to determine the resistance to crack growth of a monolithic elastoplastic triangular lattice under mode II loading. The 
displacement field associated with a remote mode II stress intensity factor $K_{\text {II }}$ is applied to the boundary nodes of the lattice, with the functions $f_{i}(\theta)$ in (7) now taken to be the corresponding functions for mode II from Sih et al. [33]. Mode II $R$-curves have been generated for a monolithic, fully triangulated lattice with $t / \ell=0.1, \varepsilon_{0 S}=0.001$ and $\bar{\Gamma}=5$. The crack growth responses are shown in Fig. 8(a) for $N=0.33$ and selected values of $\varepsilon_{\mathrm{f}}$ in the range 0.02 to 0.2. The elastic-brittle response is included in Fig. 8(a) for comparison; this is obtained by assuming a linear elastic response up to a tensile strength of magnitude $\sigma_{0 S}$ (implying $\varepsilon_{\mathrm{f}}=\sigma_{0 S} / E_{\mathrm{S}}=\varepsilon_{0 S}$ ). The sensitivity of the mode II $R$-curve to the value of the strain hardening exponent $N$ is shown in Fig. 8(b) for the choice of $\varepsilon_{\mathrm{f}}=0.1$.

For all the combinations of $\left(N, \varepsilon_{\mathrm{f}}\right)$ considered in this study, FE simulations of the crack growth in an elastoplastic lattice under mode II loading predict that the crack grows at an inclination of $-30^{\circ}$ from the plane of the pre-crack, as a series of vertical struts (labelled A in the insert of Fig. 8(a)) and inclined struts (labelled as B in the insert of Fig. 8(b)) fail under increasing remote $K_{\text {II. }}$. Inclined struts (type B) fail first. The vertical struts (type A) then bridge the crack faces, and they rotate to align with the local principal direction $\left(\theta=-30^{\circ}\right)$ until they also fail in tension. Accordingly, we show in Fig. 8(a) two $R$-curves, one corresponding to the failure of type A struts and the other corresponding to the failure of type B struts. Each cross mark in Fig. 8(a) denotes the $x_{1}$-position of the mid-point of all failed struts at a given value of $K_{\mathrm{II}}=K_{\mathrm{R}}$ with the rightmost cross-mark (corresponding to the furthest failed strut from the pre-crack tip) denoting the crack extension $\Delta a$. For the choice $N=0.33$ and $\varepsilon_{\mathrm{f}}=0.1$, the FE simulations predict that the first strut A fails after 4 struts of type $B$ have failed, see Fig. 8(a). Consequently, there is a moderate increase in the mode II fracture toughness due to bridging by the type A struts; this is evident from Fig. 8(a) for all assumed values of $\varepsilon_{\mathrm{f}}$ between 0.02 and 0.2. Curve-fit estimates of the $R$-curves corresponding to struts of type $B$ are drawn in dashed lines in Fig. 8(a) and Fig. $8(\mathrm{~b})$. These have the form

$$
K_{\mathrm{R}}=K_{\mathrm{IIC}}+\beta_{1} \bar{\rho} \sigma_{0 \mathrm{~S}} \sqrt{\ell}(\Delta a / \ell)^{\beta_{2}}
$$

where $\beta_{1}$ and $\beta_{2}$ are constants which depend upon $N$ and $\varepsilon_{\mathrm{f}}$, as listed in Table 1.

The sensitivity of the $R$-curve to strain hardening $N$ is plotted in Fig. $8(\mathrm{~b})$ for selected values of $\left(t / \ell, \varepsilon_{\mathrm{f}}\right)=(0.1,0.1)$ and $N$ between 0.1 and 0.33 . As before, separate $R$-curves are shown for type A and type B struts. Upon comparing the responses of Fig. 3 and Fig. 8 , 
we find that the crack growth resistance is only mildly sensitive to the mode of remote loading: $K_{\mathrm{R}}^{\mathrm{II}} \approx 0.8 K_{\mathrm{R}}^{\mathrm{I}}$ for a given value of crack extension $\Delta a>0$. Whilst the crack path under mode I loading is sensitive to the choice of $\left(N, \varepsilon_{\mathrm{f}}\right)$ of the cell wall solid, the crack path under remote mode II loading occurs along $\theta=-30^{\circ}$ for all combinations of $\left(N, \varepsilon_{\mathrm{f}}\right)$ assumed in this study.

We also note that the the critical mode II fracture toughness, $K_{\text {IIC }}$, associated with first strut failure is approximately of the same magnitude as the critical mode I fracture toughness $K_{\mathrm{IC}}$, for any given combination of $\left(N, \varepsilon_{\mathrm{f}}\right)$. This insensitivity of the critical fracture toughness to the mode of remote loading was observed previously in the numerical analysis of Fleck and Qiu [17] for an elastic-brittle triangular lattice. They found that the failure envelope under a combination of remote $\left(K_{\mathrm{I}}, K_{\mathrm{II}}\right)$ loading is nearly circular in shape, implying $K_{\text {IIC }} \approx K_{\text {IC }}$. The location of first strut failure is, however, sensitive to the macroscopic loading direction: under mode I loading, the first strut to fail is the vertical strut directly ahead of the crack-tip (and along the plane of the pre-crack) whereas under mode II loading, the critical strut is the strut directly ahead of the crack but inclined at $\theta=-30^{\circ}$ to the plane of the pre-crack. This finding is consistent with the observations of Fleck and Qiu [17] who noted a similar shift of the critical failure site from mode I to mode II, in an elastic-brittle triangular lattice.

\section{Concluding remarks}

FE simulations of crack growth in an elastoplastic triangular lattice suggest that an increase in fracture toughness occurs with crack extension due to non-proportional stressing of the struts within the plastic zone at the tip of the advancing crack. Additional toughening by crack bridging occurs under mode II loading. A strong dependence of the mode I crack path on $\varepsilon_{\mathrm{f}}$ is noted: for low to moderate values of cell wall failure strain $\varepsilon_{\mathrm{f}}$ such as $0.02 \leq \varepsilon_{\mathrm{f}} \leq 0.1$ the crack path resembles a triangular waveform with the amplitude and wavelength of the waveform reducing with increasing $\varepsilon_{\mathrm{f}}$. A straight-ahead mode I crack path is predicted for $\varepsilon_{\mathrm{f}}>0.1$. In contrast, the mode II crack always propagates along $\theta=-30^{\circ}$ to the plane of the macroscopic pre-crack for all combinations of material properties considered in this study. 
The presence of a ductile phase, either in the form of a large scale lattice, or in the form of longitudinal fibres transverse to the cracking plane, substantially elevates the mode I fracture toughness of the brittle phase as the ductile struts bridge the crack faces. It remains to verify these predictions experimentally. While the crack paths can be different under mode I and mode II loading for a given combination of cell wall ductility $\varepsilon_{\mathrm{f}}$ and strain hardening exponent $N$, the $R$-curves are only mildly sensitive to the mode of loading. Again, experiments are warranted to confirm (or refute) this finding.

\section{Acknowledgements}

The authors gratefully acknowledge the financial support from the European Research Council (ERC) under the European Union's Horizon 2020 research and innovation program, grant GA669764, MULTILAT. The authors are also deeply grateful to the reviewers for their valuable comments and helpful suggestions. 


\section{References}

[1] A. G. Evans, J. W. Hutchinson, N. A. Fleck, M. F. Ashby, H. N. G. Wadley, The Topological Design of Multifunctional Cellular Metals, in: Progress in Materials Science, vol. 46, 309-327, 2001.

[2] H. N. G. Wadley, N. A. Fleck, A. G. Evans, Fabrication and Structural Performance of Periodic Cellular Metal Sandwich Structures, Comp. Sci. Tech. 63 (2003) 2331-2343.

[3] H. N. G. Wadley, Multifunctional Periodic Cellular Metals, Phil. Transac. Series A 364 (2006) 31-68.

[4] D. Mahmoud, M. Elbestawi, Lattice structures and functionally graded materials applications in additive manufacturing of orthopedic implants: a review, Journal of Manufacturing and Materials Processing 1 (2) (2017) 13.

[5] B. Jenett, C. Gregg, D. Cellucci, K. Cheung, Design of multifunctional hierarchical space structures, in: 2017 IEEE Aerospace Conference, IEEE, 1-10, 2017.

[6] S. Govender, H. Friedrich, Monoliths: a review of the basics, preparation methods and their relevance to oxidation, Catalysts 7 (2) (2017) 62.

[7] E. Del Olmo, E. Grande, C. Samartin, M. Bezdenejnykh, J. Torres, N. Blanco, M. Frovel, J. Caas, Lattice structures for aerospace applications, European Space Agency, (Special Publication) ESA SP 691.

[8] L. Montanaro, A. Bachiorrini, A. Negro, Deterioration of cordierite honeycomb structure for diesel emissions control, Journal of the European Ceramic Society 13 (2) (1994) 129 - 134, ISSN 0955-2219.

[9] L. Dong, V. Deshpande, H. Wadley, Mechanical response of Ti-6Al-4V octet-truss lattice structures, Int. J. Solids Struct. 60-61 (2015) 107 - 124.

[10] T. Tancogne-Dejean, A. B. Spierings, D. Mohr, Additively-manufactured metallic microlattice materials for high specific energy absorption under static and dynamic loading, Acta Materialia 116 (2016) $14-28$.

[11] L. J. Gibson, M. F. Ashby, Cellular Solids: Structure and Properties, Cambridge University Press, Cambridge, 1997. 
[12] N. Romijn, N. Fleck, The fracture toughness of planar lattices: Imperfection sensitivity, J. Mech. Phys. Solids 55 (2007) 2538-2564.

[13] S. N. Khaderi, V. S. Deshpande, N. A. Fleck, The stiffness and strength of the gyroid lattice, Int. J. Solids Struct. .

[14] H. C. Tankasala, V. S. Deshpande, N. A. Fleck, Crack kinking at the tip of a mode I crack in an orthotropic solid, Int. J. Frac. 82 (9) (2017) 1-10.

[15] D. D. Symons, J. Shieh, N. A. Fleck, Actuation of the Kagome Double Layer Grid. Part 2: Effect of Imperfections on the Measured and Pre- dicted Actuation Stiffness, J. Mech. Phys. Solids 53 (2005) 1875-1891.

[16] P. E. Seiler, H. C. Tankasala, N. A. Fleck, The role of defects in dictating the strength of brittle honeycombs made by rapid prototyping, Acta Materialia 171 (2019) 190 - 200.

[17] N. A. Fleck, X. Qiu, The damage tolerance of elastic-brittle, two-dimensional isotropic lattices, J. Mech. Phys. Solids 55 (2007) 562-588.

[18] J. L. Grenestedt, Influence of Wavy Imperfections in Cell Walls on Elastic Stiffness of Cellular Solids, J. Mech. Phys. Solids 46 (1998) 29-50.

[19] Q. I. Alonso, N. A. Fleck, Fracture of Brittle Lattice Materials : A Review, in: I. M. Daniel, E. E. Gdoutos, Y. D. S. Rajapakse (Eds.), Major accomplishments in Composite materials and sandwich structures - An anthology of ONR sponsored research, Springer, 799816, 2009.

[20] M. R. O'Masta, L. Dong, L. St-Pierre, H. N. Wadley, V. S. Deshpande, The fracture toughness of octet-truss lattices, J. Mech. Phys. Solids 98 (2017) 271-289.

[21] H. Gu, M. Pavier, A. Shterenlikht, Experimental study of modulus, strength and toughness of 2D triangular lattices, International Journal of Solids and Structures 152-153 (2018) $207-216$.

[22] H. C. Tankasala, V. S. Deshpande, N. A. Fleck, 2013 Koiter Medal Paper: Crack-Tip Fields and Toughness of Two-Dimensional Elastoplastic Lattices, J. Appl. Mech. 82 (9) (2015) 1-10. 
[23] P. R. Onck, R. Van Merkerk, J. T. M. De Hosson, I. Schmidt, Fracture of Metal Foams: In-Situ Testing and Numerical Modeling, Adv. Engg. Mat. 6 (6) (2004) 429-431.

[24] K. R. Mangipudi, P. R. Onck, Notch Sensitivity of Ductile Metallic Foams: A Computational Study, Acta Mat. 59 (19) (2011) 7356-7367.

[25] K. R. Mangipudi, P. R. Onck, Multiscale modelling of damage and failure in twodimensional metallic foams, J. Mech. Phys. Solids 59 (7) (2011) 1437-1461.

[26] N. E. de Kruijf, R. H. J. Peerlings, M. G. D. Geers, An Analysis of Sheet Necking under Combined Stretching and Bending, International Journal of Material Forming 2 (2009) 845-848.

[27] V. Tvergaard, J. W. Hutchinson, The relation between crack growth resistance and fracture process parameters in elastic-plastic solids, J. Mech. Phys. Solids 40 (6) (1992) 1377-1397.

[28] C. Chen, N. A. Fleck, T. J. Lu, The Mode I Crack Growth Resistance of Metallic Foams, J. Mech. Phys. Solids 49 (2001) 231-259.

[29] I. Schmidt, N. A. Fleck, Ductile Fracture of Two-Dimensional Cellular Structures, Int. J. Frac. 111 (2001) 327-342.

[30] L. R. Meza, G. P. Phlipot, C. M. Portela, A. Maggi, L. Montemayor, A. Comella, D. M. Kochmann, J. R. Greer, Reexamining the mechanical property space of threedimensional lattice architectures, Acta Materialia 140 (2017) 424 - 432.

[31] V. S. Deshpande, M. F. Ashby, N. A. Fleck, Foam topology bending versus stretching dominated architechtures, Acta Mat. 49 (2001) 1035-1040.

[32] N. A. Fleck, V. S. Deshpande, M. F. Ashby, Micro-Architectured Materials: Past, Present and Future, Proc. Royal Soc. A 466 (5) (2010) 2495-2516.

[33] G. Sih, P. Paris, G. Irwin, On cracks in rectilinearly anisotropic bodies, Int. J. Frac. Mech. 3 (1965) 189-203.

[34] Y. F. Gao, A. F. Bower, A simple technique for avoiding convergence problems in finite element simulations of crack nucleation and growth on cohesive interfaces, Modelling and Simulation in Materials Science and Engineering 12 (3) (2004) 453-463. 
[35] J.-Y. Sun, X. Zhao, W. R. Illeperuma, O. Chaudhuri, K. H. Oh, D. J. Mooney, J. J. Vlassak, Z. Suo, Highly stretchable and tough hydrogels, Nature 489 (7414) (2012) 133-136. 
Tables

\begin{tabular}{lccccccc}
\hline$N$ & $\varepsilon_{\mathrm{f}}$ & $\alpha_{1}$ & $\alpha_{2}$ & $\frac{K_{\mathrm{IC}}}{\sigma_{0 S} \sqrt{\ell}}$ & $\beta_{1}$ & $\beta_{2}$ & $\frac{K_{\text {IIC }}}{\sigma_{0 S} \sqrt{\ell}}$ \\
\hline Elastic-brittle & - & 0.66 & 0.04 & 0.21 & 0.06 & 0.25 & 0.23 \\
0.1 & 0.1 & 3.17 & 0.43 & 2 & 0.72 & 0.36 & 2 \\
0.2 & 0.1 & 3.55 & 0.5 & 3.2 & 0.98 & 0.6 & 2.6 \\
0.33 & 0.02 & 1.44 & 0.32 & 1.35 & 0.69 & 0.46 & 0.7 \\
0.33 & 0.05 & 2.68 & 0.39 & 2.4 & 0.83 & 0.54 & 2 \\
0.33 & 0.1 & 4.47 & 0.48 & 3.6 & 1.12 & 0.67 & 3.2 \\
0.33 & 0.2 & 6.93 & 0.55 & 6 & 2.02 & 0.74 & 6 \\
\hline
\end{tabular}

Table 1: Values of the constants in the curve-fit expression Eq. (11) for mode I $R$-curves plotted in Fig. 3 and values of the constants in expression Eq. (13) for mode II $R$-curves (based on failure of type B struts) plotted in Fig. 8. 


\section{Figures}

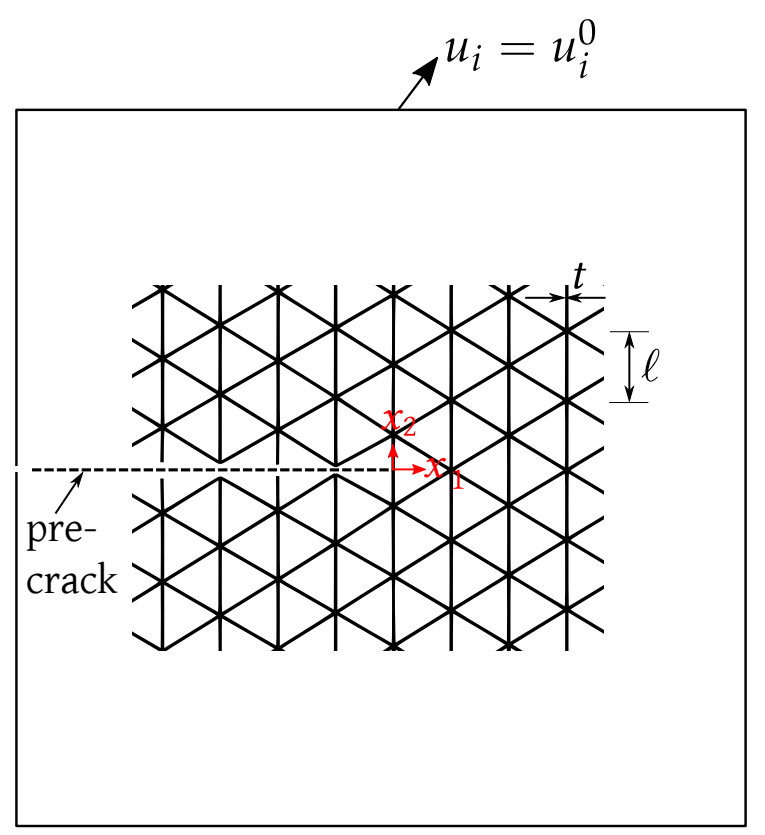

Fig. 1: Triangular lattice containing a semi-infinite crack and subjected to remote mode I (or mode II) loading

(a)

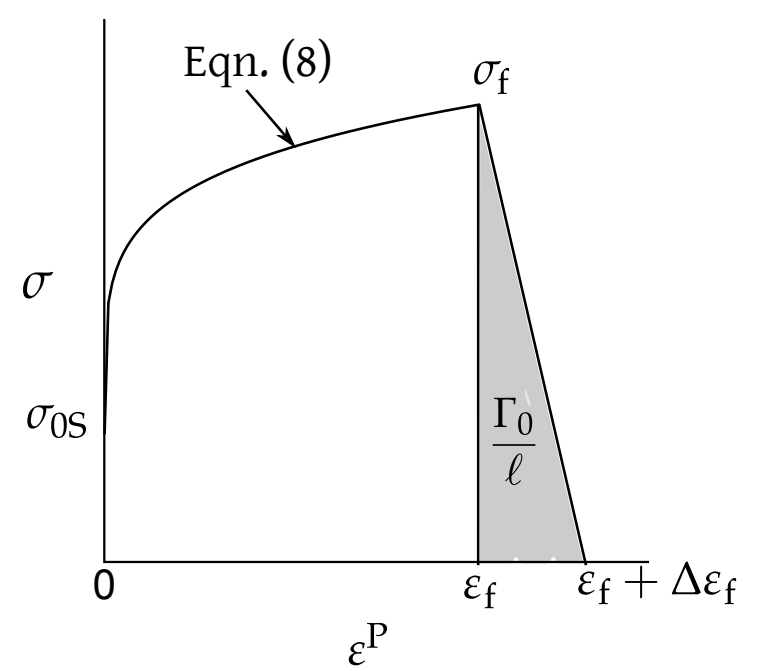

(b)

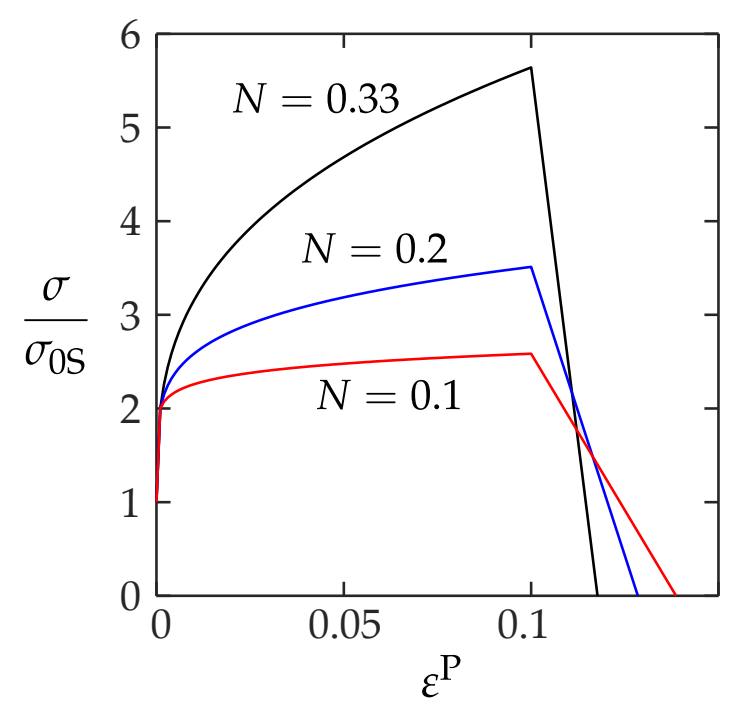

Fig. 2: Stress versus plastic strain response of a single strut in tension: (a) material parameters employed in the continuum damage model for each strut, and (b) response for $N=0.1,0.2$, and 0.33 for the choice of $\varepsilon_{\mathrm{f}}=0.1$ and $\bar{\Gamma}=\Gamma_{0} E_{\mathrm{S}} / \sigma_{0 \mathrm{~S}}^{2} \ell=50$. 
(a)

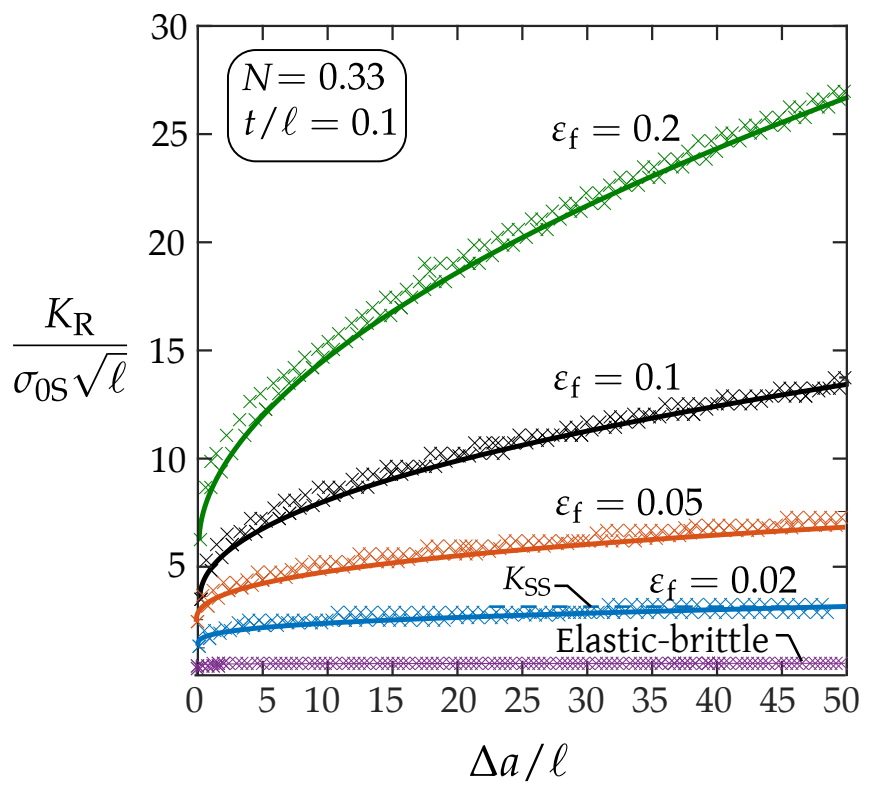

(c)

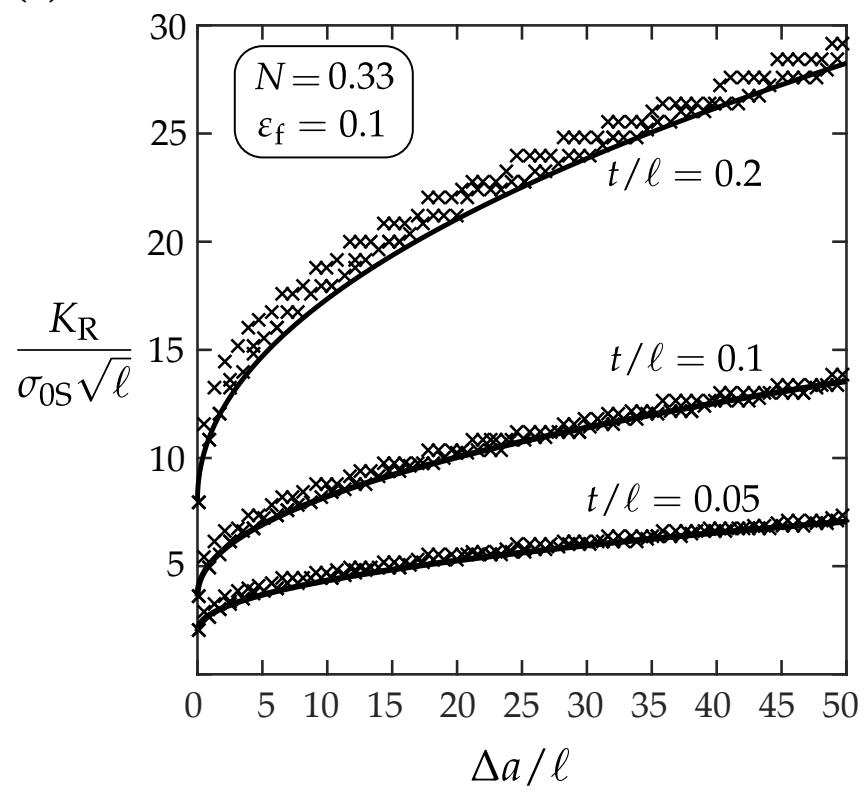

(b)

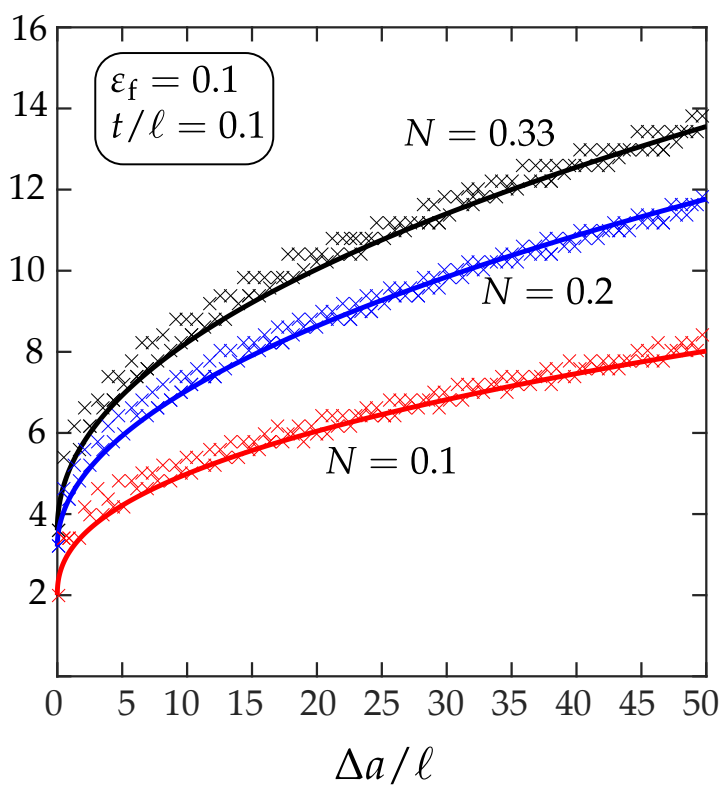

(d)

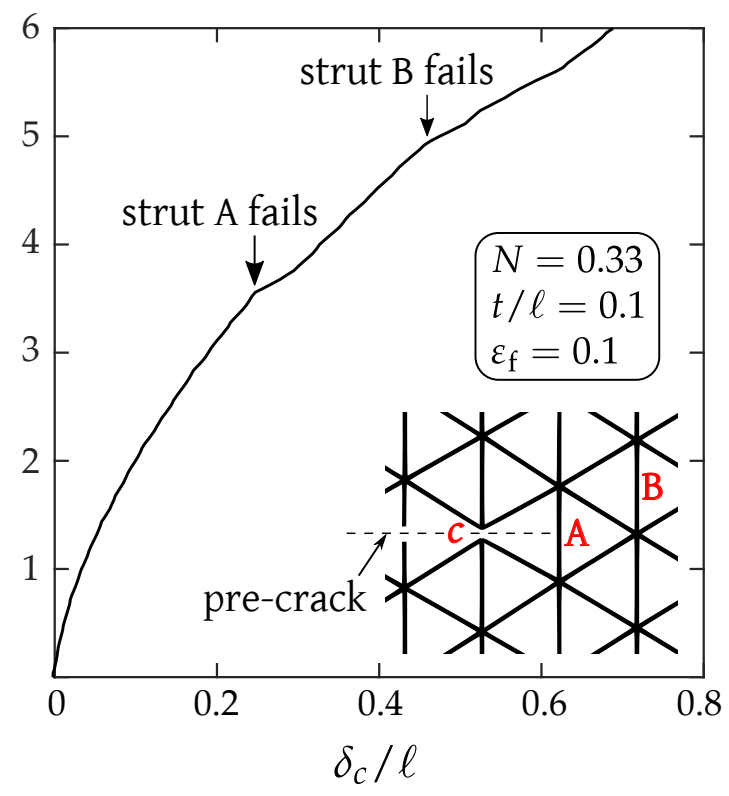

Fig. 3: Mode I crack growth resistance of an elastoplastic triangular lattice : (a) as a function of strut ductility $\varepsilon_{\mathrm{f}},(\mathbf{b})$ as a function of the strain-hardening exponent $N$, and (c) as a function of lattice relative density $\bar{\rho}$ expressed in terms of $t / \ell$ via (1). In all cases, $\varepsilon_{0 S}=0.001$ and $\bar{\Gamma}=5$. (d) The dependence of crack-tip opening displacement $\delta_{c}$ upon $K_{\mathrm{R}}$ in the early stages of crack advance. 

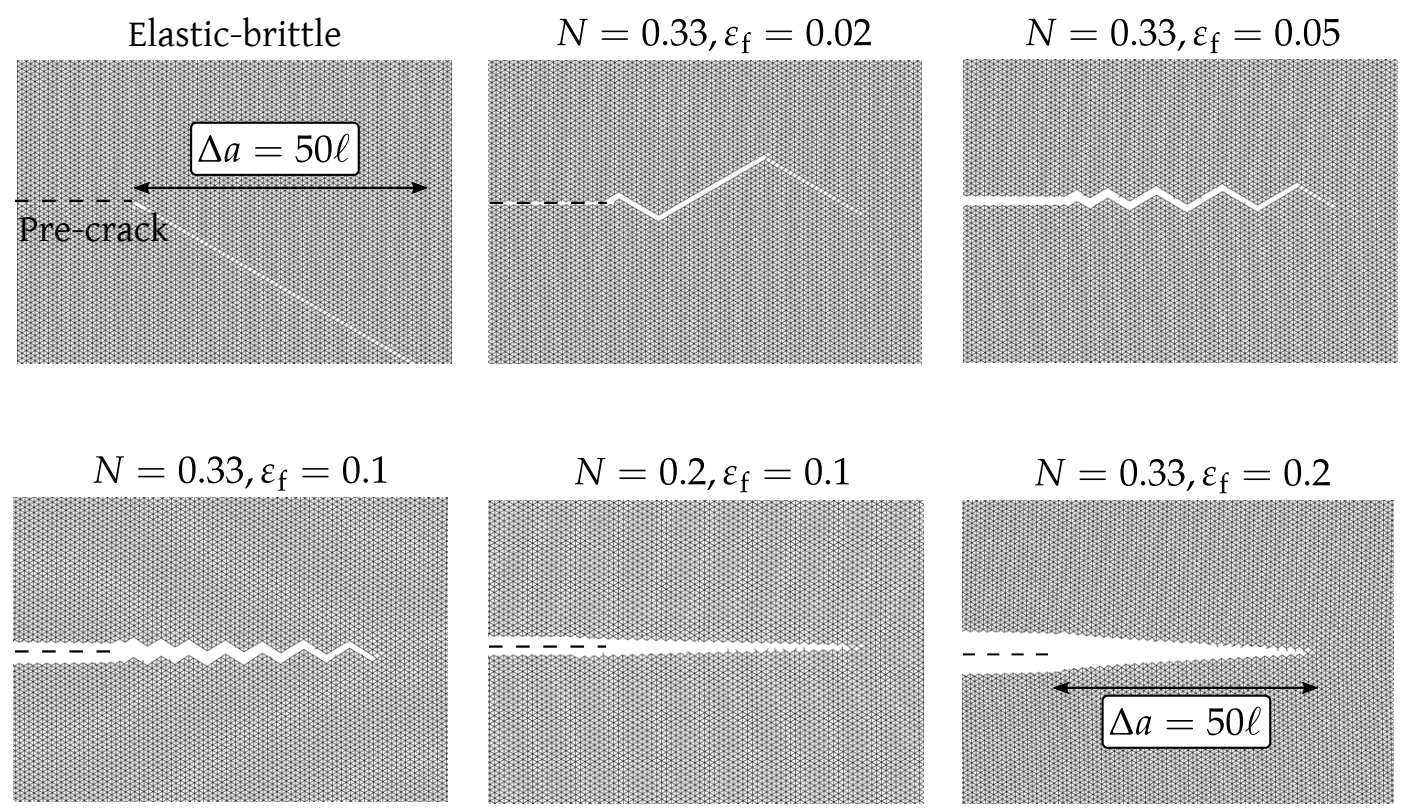

Fig. 4: Crack path under remote mode I loading as a function of strut ductility $\varepsilon_{\mathrm{f}}$ and strainhardening exponent $N$. In all cases, $\varepsilon_{0 S}=0.001, t / \ell=0.1$, and $\bar{\Gamma}=5$.

(a)

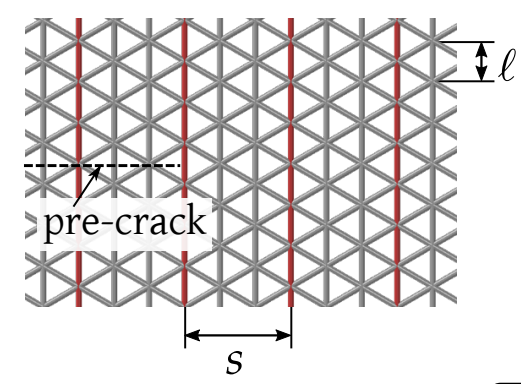

(b)

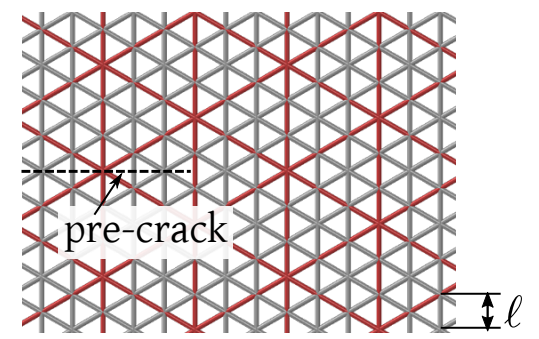

(c)

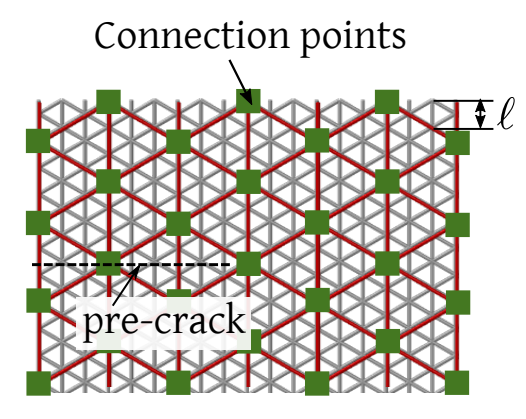

\section{Brittle phase - Ductile phase}

Fig. 5: Two-phase lattices: (a) Fibre-reinforced triangular lattice, (b) Triangular bilattice, and (c) Interpenetrating triangular lattice. 
(a)

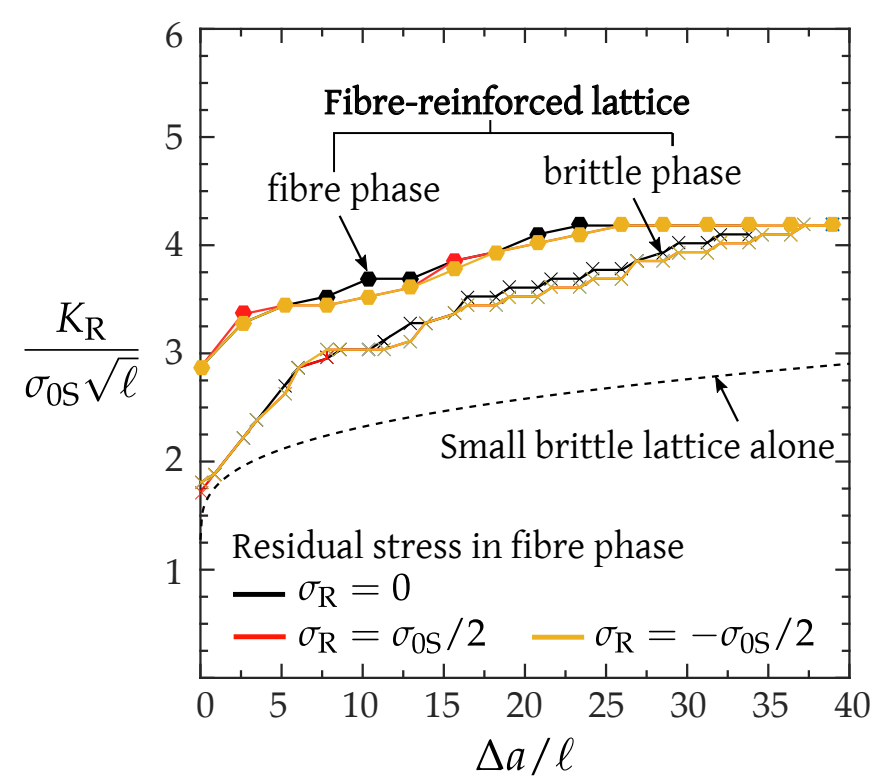

(b)

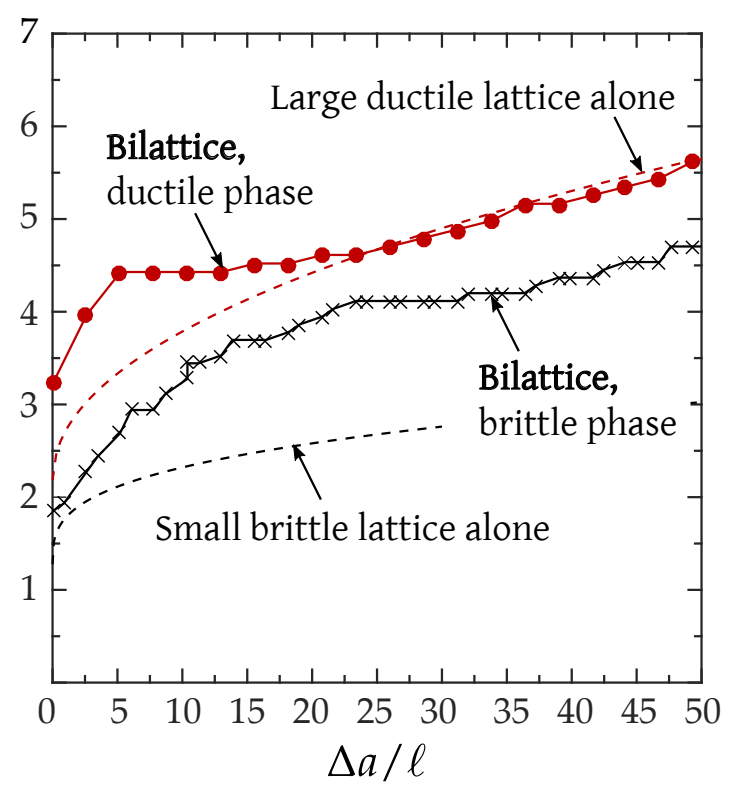

(c)

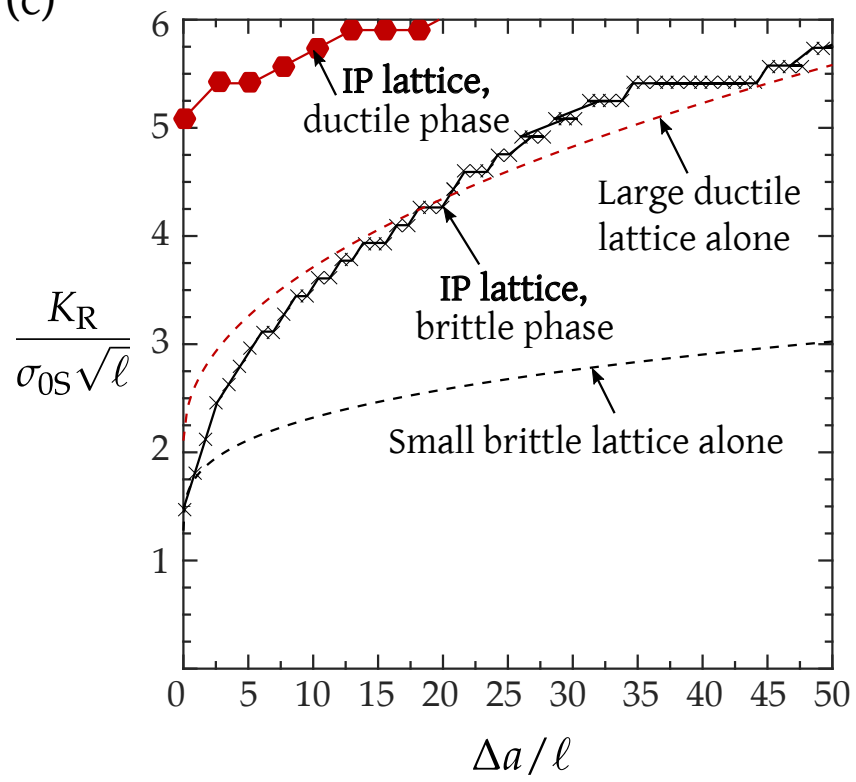

Fig. 6: $R$-curves of the two-phase lattices: (a) fibre-reinforced lattice, (b) bilattice, and (c) interpenetrating (IP) triangular lattice. The $R$-curve for the brittle lattice is included in (a), and the $R$-curves for both the small brittle and large ductile lattices are shown in (b) for comparison. In all cases, $t / \ell=0.1, N=0.33, \varepsilon_{\mathrm{f}}=0.02$ for the brittle phase and $\varepsilon_{\mathrm{f}}=0.1$ for the ductile phase. Data points in (a)-(c) correspond to the location of the mid-points of the failed vertical struts. 
(a)

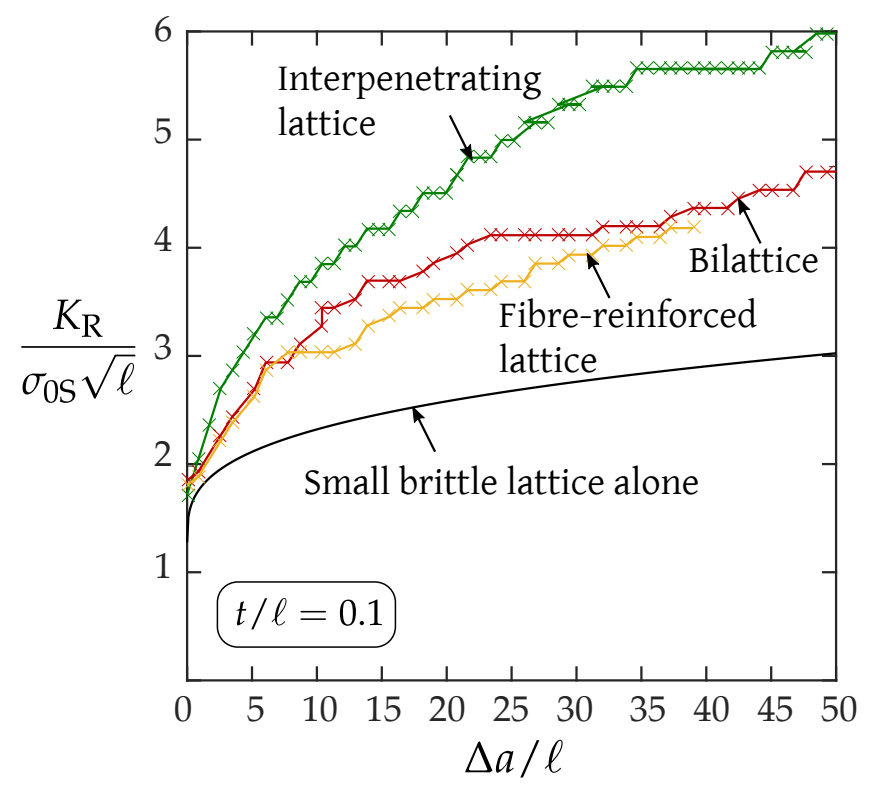

(b)

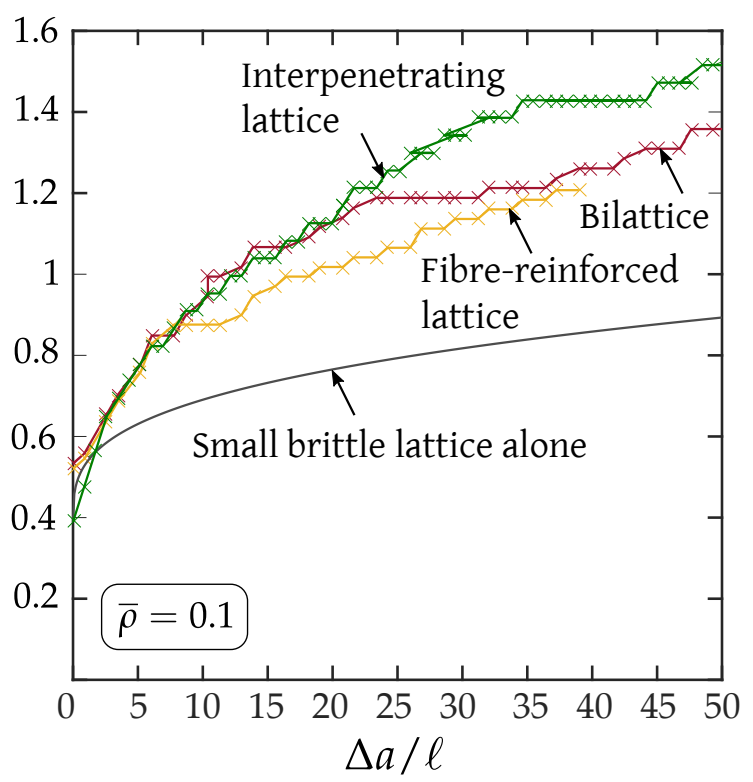

Fig. 7: Toughening of a brittle lattice by the addition of a ductile phase: (a) for a constant $t / \ell=0.1$, and (b) for a constant $\bar{\rho}=0.1$ of the two-phase lattice. In all cases, $N=0.33$ and $\varepsilon_{\mathrm{f}}=0.02$ for the brittle phase. 
(a)
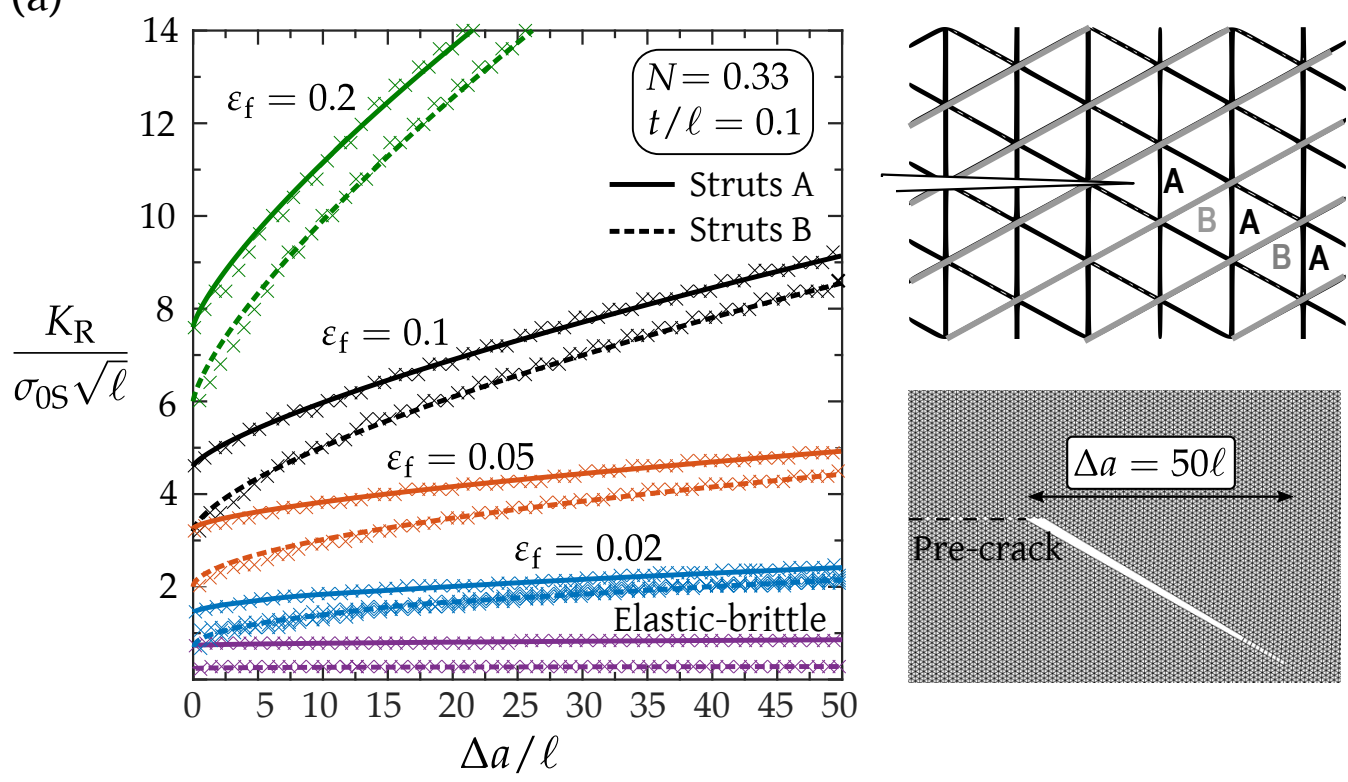

(b)

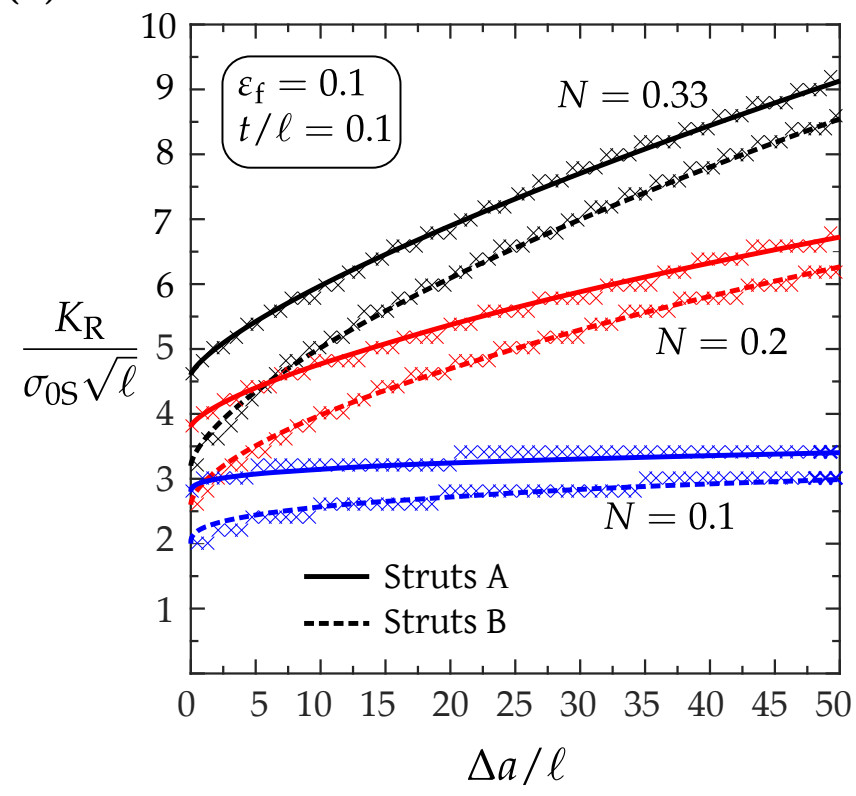

Fig. 8: Mode II crack growth resistance of an elastoplastic triangular lattice : (a) as a function of strut ductility $\varepsilon_{\mathrm{f}}$ for a choice of $N=0.33$, and (b) as a function of the strainhardening exponent $N$ for a choice of $\varepsilon_{\mathrm{f}}=0.1$. In all cases, $\varepsilon_{0 \mathrm{~S}}=0.001, t / \ell=0.1$, and $\bar{\Gamma}=5$. Inset of (a) shows predicted crack path at $\Delta a=50 \ell$ for crack growth under remote mode II loading. 